\title{
ARTIGOS
}

\section{CONTAR HISTORIAS: HEIDEGGER Y DERRIDA PENSADORES DE LA HISTORICIDAD*}

\author{
Andrea Potestà ${ }^{* *}$ \\ apotesta@uc.cl
}

RESUMO $O$ artigo tem por objetivo analisar a raiz heideggeriana do tratamento da história no jovem Derrida. Juntamente à sua análise do problema genético em Husserl, Derrida constrói sua discussão crítica da razão histórica referindo-se ao problema da historicidade originária em "Ser e Tempo". São conhecidos os seus estudos dos anos 1970 em matéria de Heidegger, em torno do problema do tempo e da diferença (em "Ousia et grammé" e no ensaio sobre "La différance", por exemplo), porém, uma nova e mais explícita evidência sobre esta relação primária com Heidegger em torno do problema histórico é oferecida pelo curso que Derrida ministrou na École Normale Supérieure de Paris no período 1964-1965, sob o título "Heidegger: a questão do Ser e da História". Nesse curso, que acaba de ser publicado, Derrida discute a questão linguística associando-a ao problema heideggeriano da contaminação ineludivel entre narração e fundação. No texto que segue, pretende-se analisar os termos de tal herança heideggeriana e, ao mesmo tempo, destacar as questões críticas que emergem desse curso e detalham a distância entre o jovem Derrida e Heidegger.

Palavras-chave Historicidade, Heidegger, Derrida, narração, ficção.

ABSTRACT The article aims at analyzing the treatment of history in Young Derrida under a Heideggerian basis. Together with that, add Heidegger's analysis on the genetic issue in Husserl, Derrida builds his critical discussion

* Este texto es parte del proyecto Fondecyt n. 1160479.

** Universidad Católica de Chile. Artigo recebido em 21/03/2016 e aprovado em 29/06/2016. 
on the historical reason referring to the originary historicity issue in "Ser e Tempo". His studies from the 70s, in terms of Heidegger, are known, regarding the issue of time and difference (in "Ousia et gramme" and in the essay on "La difference", for example), but, a new and more explicit evidence on that primary relation with Heidegger round the historical issue is offered by the course Derrida taught at the École Normale Supérieure de Paris from 19641965, under the title "Heidegger: a questão do Ser e da História". In that course, which has just been published, Derrida discusses the linguistic issue linking it to Heideggerian issue of the unavoidable contamination between narrations and foundation. In the text below, we intend to analyze terms such as Heideggerian heritage and, at the same time, highlighting the critical issues emerging from that course and which detail the distance between the young Derrida and Heidegger.

Keywords Historicity, Heidegger, Derrida, narration, fiction.

\author{
Aux historiens. \\ Soyez juges. Soyez apôtres. Soyez prêtres. \\ Dites le vrai. Surtout n'expliquez pas les traîtres! \\ Car l'explication finit par ressembler \\ A l'indulgence affreuse; et cela fait trembler. \\ $\mathrm{Ne}$ me racontez pas un opprobre notoire \\ Comme on raconterait n'importe quelle histoire. \\ Victor Hugo (1888, p. 184)
}

\title{
Introducción
}

El tema de la historicidad es por cierto clave en la propuesta deconstructiva. Desde la cuestión abierta en la "Introducción a El origen de la geometría" con la que se discute Husserl a la luz del problema de la historicidad de los objetos ideales, hasta temas como el de la tradición, de la herencia, o del archivo, siempre se corrobora una atención de Derrida por la problemática histórica. El esquema de tal problemática se define, quizás de la manera más completa, en la confrontación de Derrida con Husserl, en la que Derrida muestra temáticamente el problema de la alteridad temporal que parasita el presente vivo, llegando así a comprender el tema de la historia a la luz del problema diferencial. Sin embargo, luego de la discusión temática con Husserl, existe el aporte crítico 
heideggeriano del que Derrida ha sacado seguramente mucho provecho ya en sus primeros pasos. En el 2013, se ha publicado en Francia un documento que permite apreciar esta centralidad de modo genético. Se trata del curso que Derrida ha impartido en la École Normale Supérieure de Paris en el 1964-1965 bajo el título "Heidegger: la cuestión del Ser y de la Historia", en el que se realiza el paso inaugural y decisivo de la lectura derridiana de Heidegger. Como ha sido ya observado (Cf. Naas, 2015; Gratton, 2015), Derrida discute en este curso con Heidegger (y con Hegel) buscando en la historicidad los elementos clave de un giro filosófico capaz de asumir la complejidad del problema lingüístico y la contaminación ineludible entre narración y fundación.

El texto que sigue pretende analizar el problema de tal contaminación y redefinir el terreno común entre Heidegger y Derrida en el ámbito histórico. Se procederá, primero, indicando algunos pliegues fundamentales del gesto heideggeriano sobre la historicidad en general, haciendo una alusión al tratamiento que le reserva Heidegger en "Ser y tiempo" (texto del que se ocupa principalmente el curso de Derrida). En un segundo momento, se precisarán los elementos que Derrida subraya en su lectura, insistiendo en particular en la cuestión de la narración y de la circularidad hermenéutica, y se mostrará de qué forma Derrida intenta valorizar cierta duplicidad del tratamiento heideggeriano de la historia. Finalmente, el artículo pretende esbozar los elementos de una oposición a Heidegger que emerge en ese curso de modo apenas perceptible, pero no por esto menos insistente, y que constituye la base de las ulteriores discusiones de Derrida con Heidegger. Se trata entonces de poner de relieve y de reconstruir con esta lectura los primeros pasos del joven Derrida frente a Heidegger para entender la génesis del tema diferencial en su derivación heideggeriana.

\section{Acontecer}

Si pudiéramos conocer los acontecimientos en sí mismos, los hechos brutos en su pureza incondicional y elemental, la historia sería una ciencia absoluta (probablemente la única ciencia, capaz de definir cualquier cosa exhaustivamente y para siempre) y su objeto sería la estructura misma del sentido. Pero este axioma (al que apunta, por supuesto, la dialéctica hegeliana) choca con una evidencia a su vez absoluta: el hecho de que las estructuras del sentido con las que se capta el sentido mismo de la historia son, ellas mismas, históricas. O sea, el modo con el que se recibe un hecho, o con el que se lo cuenta, construyendo una narración más o menos factual -o, en términos más simples, el modo en el que se lo piensa, dándole un orden, un inicio y un fin, 
una orientación inteligible-, no escapa a su vez de la factualidad y no sale del devenir histórico. No se puede por lo tanto alcanzar una historia única y pura, como ya el Nietzsche de las "Consideraciones intempestivas" había observado: todo historicismo que pretenda aplicar mallas de lectura universales de los hechos ya insinúa una instancia no-histórica en lo histórico, dogmatizando inexorablemente el espacio de su sentido y llegando a esa "inhibición vital" que, según Nietzsche, conduce "al deterioro y a la degeneración de la vida" (Nietzsche, 2006, p. 10).

Más allá de Nietzsche, hay por supuesto toda una tradición de pensadores que, desde comienzos del siglo pasado, se han dado a la tarea de repensar en profundidad la historia. Junto a otros gestos, por cierto, revolucionarios -me refiero para empezar al de Wilhelm Dilthey-, está, como es sabido, el intento de Martin Heidegger. Heidegger es probablemente quien ha sabido llevar ese problema de la forma más radical a sus últimas consecuencias, o por lo menos, dar al problema de la historia una centralidad que le hizo necesario extenderlo a todos los demás campos del pensamiento. Heidegger reconsidera el problema mismo del sentido (del ser) a la luz de la facticidad mundana e histórica del Dasein, del existente, que solo desde su existir concreto y diseminado en el mundo del acontecer -es decir, afuera de toda consideración "estructural" y egóica, trascendente y centrada- puede valorar el sentido en su efectivo acontecer dinámico. Heidegger es el que más ha insistido en radicalizar este propósito, esta preocupación, en la dirección abierta por su ontología y por su analítica de la existencia.

Para Heidegger es necesario considerar la existencia del Dasein en su diseminación concreta y aconteciente, porque esto ofrece la garantía de no estar encerrando al Dasein dentro de una constitución metahistórica o extrahistórica, o sea, en la jerga de "Ser y tiempo", "categorial". Heidegger quiere plantear el problema del ser y pensarlo a la luz de la cuestión del tiempo, pero para hacer esto es necesario, primero, encarnar históricamente la pregunta en la situación de la existencia fáctica. Este es el sentido de la "etapa preparatoria" anunciada en "Ser y tiempo" (Heidegger, 1997, p. 50). Con las palabras de Heidegger: "se requiere una explicación originaria del tiempo como horizonte de la comprensión del ser a partir de la temporeidad en cuanto ser del Dasein comprensor del ser" (ibid., p. 28).

Debemos entonces empezar por la historicidad concreta del Dasein fáctico, si queremos ganar el horizonte para una interpretación del ser en general, evitando así el error de la metafísica que olvida el carácter aconteciente del sentido del ser. 
Es en el capítulo 5 de la segunda (y última) sección de "Ser y tiempo" que Heidegger desarrolla el tema de la "historia aconteciente", que debería permitirle una entrada en la auténtica temporalidad del propio Dasein (opuesta a la comprensión vulgar del tiempo o a una historia de meros hechos). El tema es de suma importancia en la economía de la analítica heideggeriana, en la medida en que, como se dice en el $\S 76$, "la tematización primaria del objeto del saber histórico proyecta al Dasein que ha-existido hacia su más propia posibilidad de existencia" (ibid., p. 379).

Dicho de otro modo, es solo al realizar un análisis de la trama vital que tiene lugar entre nacimiento y muerte del Dasein y al problematizar existencialmente ese "entre", que será posible considerar el modo en que el Dasein acontece factualmente, y de ahí tener una comprensión de la trama histórica fundamental de la existencia. ¿Y cómo acontece el Dasein? El Dasein acontece prolongándose como sido, presente y futuro, o sea, acontece en función de determinaciones existenciales: el Dasein "se asume" y "opta por...". Se asume como pasado y como tradición, en el modo de la repetición, y "opta por...", como proyecto futuro, en la medida de su anticipación de la muerte. Esta complejidad en su conjunto retentivo-proyectivo es el acontecer histórico del Dasein, esto es, su propia constitución existencial en términos temporales que, como dice Heidegger, hace al Dasein libre para devenir en su finitud un destino histórico.

No es necesario adentrarse aquí en todos los argumentos explícitos de Heidegger sobre las determinaciones de ese "destino" y toda la carga ideológica que se le asocia, que por supuesto ha sido el objeto de varias relecturas críticas; ${ }^{1}$ vale la pena en cambio limitarse al esquema general del argumento heideggeriano. Es lo que hace de hecho Derrida en su curso del 1964-65. El esquema general del argumento heideggeriano es muy sencillo: hay que evitar caer en el error de la tradición metafísica, evitar pensar la historia como Historie, como lo hace la historiografía clásica, que se refiere al conjunto de "hechos" estudiables y objetivables, y hay, al contrario, que entrar en el modelo de la Geschichte, la historia pensada a partir del geschehen, del acontecer existencial. O sea, se trata para Heidegger de evitar todos los riesgos de una apropiación metafísica y categorial del tema de la historia, para engendrar otra entrada.

Vale la pena insistir en esto porque después será relevante por la lectura de Derrida: se trata en Heidegger de evadir esos riesgos, y así mantener una visión perfectamente liberada de toda contaminación metafísica. En esto se expresa por lo tanto lo propio de su gesto en "Ser y tiempo": debemos buscar un modo 
autentico y apropiado de plantear el tema histórico, para salir del error. Un modo para hacer de la historicidad un tema, pero sin que esto implique una esencialización de la historia. Debemos plantear el tema de tal manera que se suspenda todo prejuicio metahistórico: con esto llegaremos a la esencialidad de la historia, a un nuevo concepto de la historia o de la historicidad, que libera la historia de los conceptos, o de las esencias. De esto se trata: de des-esencializar o des-conceptualizar la historia, para poder capturar la esencia y el concepto de la historia de modo nuevo; de salir de la visión estática y estructural de la historia, pero sin abandonarse por esto a las oscuridades de los acontecimientos brutos. Heidegger lo dice así en un momento, en el $\S 75$ :

Constantemente la interpretación existencial de la historicidad del Dasein, sin advertirlo, se sume en la oscuridad. Las oscuridades son difíciles de disipar por cuanto no se han distinguido aún las posibles dimensiones del cuestionamiento adecuado y porque en todas ellas ronda el enigma del ser y -como ahora se nos ha hecho claro- el del movimiento. Es posible, sin embargo, esbozar un proyecto de la génesis ontológica de la historia como ciencia, a partir de la historicidad del Dasein (ibid., p. 377).

Si damos la vuelta existencial, si seguimos la comprensión “desesencializante" de la existencia, y si nos preocupamos únicamente de la historicidad en tanto flujo vivencial, en tanto "entre" entre nacimiento y muerte, en tanto heredar/repetir-decidir-proyectar/dominar (que son las determinaciones con la que el Dasein se abre a su apropiación histórica), será entonces posible invertir los conceptos filosófico tradicionales y metafísicos de la historia, y marcar una distancia constitutiva, un salto en otra concreción de la historicidad.

\section{Cuento}

Este es el esquema del argumento heideggeriano. Se trata de un esquema dual: un modo es insuficiente porque reduce lo concreto a lo estructural; el otro modo se supone en cambio capaz de asumir toda la complejidad de la historia como concretitud y de la historia como estructura.

Ahora bien, en el curso del 1964-65, Derrida vuelve a pensar este argumento con una conceptualidad diferente, una conceptualidad que le permite al mismo tiempo valorizar lo propio del intento heideggeriano y mostrar un límite constitutivo de su planteamiento. Derrida retoma la expresión con la que Husserl, en las "Ideas I", marcaba la exigencia de un retorno a lo primitivo. Husserl decía en la primera nota del texto: "Es werden hier keine Geschichten erzählt" (Husserl, 1962, p. 17 nota). ¡No se cuentan historias aquí! Lo cual significaba para Husserl, que debemos tomar en serio el trabajo genético anunciado. Derrida reutiliza esta declaración de intenciones husserliana para indicar lo propio del 
gesto heideggeriano: lo que quiere Heidegger es esencialmente que paremos de "contar historias" acerca de la historia. Es como si Heidegger dijera: ¡aquí, en "Ser y tiempo" no se cuentan historias, sino que se estudia la historia tal como concierne a la situación fáctica concreta!

Obsérvese que la expresión francesa "raconter des histoires" de modo análogo al alemán "Geschichten erzählen", tiene algo peculiar que no filtra enteramente ${ }^{2}$ en castellano: significa a la vez lo que dice literalmente, o sea "contar historias", realizar un cuento narrativo relativo a un acontecimiento, pero también significa, por extensión, "mentir" o "mentirse a sí mismo": si alguien "se cuenta una historia", esto significa en francés y en alemán que está dando una explicación de mala fe o de pura conveniencia; por ejemplo es el caso de alguien que se miente a sí mismo porque necesita creer en su cuento para ser aceptado o reconocido por otros.

Ahora, Derrida observa que Heidegger quiere con su gesto salir de todo esto: quiere que no sigamos mintiéndonos sobre la historia. La historia no puede ser discutida seriamente dentro de una consideración temático-objetiva, porque ésta última "se la cree" demasiado y pervierte así el hecho concreto transformándolo en dato puro. Si alguien cree en la historia es porque acepta un cuento que se ha construido para proyectar cierto dominio sobre los acontecimientos. Heidegger quiere, al contrario, que entremos en una consideración de la historicidad capaz de suspender toda estructura fija de conceptualización (lo no-histórico, es decir, lo "mentiroso") y que se piense la historia históricamente, manteniéndose cerca del acontecer concreto y fáctico, y sin "armar cuentos", más o menos articulados, sobre él, que terminan siempre implicando estructuras inadecuadas (es decir, categoriales).

Por esto mismo, según Derrida, el objetivo crítico general de la propuesta heideggeriana sería por excelencia Hegel. Hegel pertenece a la tradición que Heidegger quiere destruir con su gesto, e incluso sería su mayor representante, al haber determinado la historia como epifanía de lo espiritual-subjetivo, es decir, de lo conceptual. Por lo tanto, como observa Derrida en el curso del 6465, "la destrucción de la historia de la ontología es también una destrucción del hegelianismo [la destruction de l'histoire de l'ontologie est aussi une destruction du hégélianisme]" (Derrida, 2013, p. 33). Debe ser así. Heidegger debe luchar con Hegel si quiere "parar de contar historias", porque es Hegel el que más ha hecho de la historia un cuento: es suficiente pensar en la construcción de la 
Fenomenología del espíritu para ver ahí la coincidencia entre historia y novela o cuento o narración conceptual.

Y para Heidegger está claro que, dicho con las palabas de Derrida:

La escritura [...] cuenta historias, la narración es fácil y la filosofía, no obstante las apariencias, no se ha nunca liberado de ella. Se trata en cambio [para Heidegger] de romper con la novela filosófica, y de romper con ella radicalmente y no para dar lugar a alguna otra novela [L'écriture [...] raconte des histoires, la narration est facile et la philosophie, malgré les apparences, ne s'en est jamais privée. Il s'agit de rompre avec le roman philosophique, et de rompre avec lui radicalement et non pour donner lieu à quelque nouveau roman] (Derrida, 2013, p. 57).

Debemos salir de la narración. Parar de "contar historias", parar de mentirnos y de construir visiones ficcionales que no podrán nunca satisfacer a los hechos de modo exhaustivo, siendo ellas mismas históricas.

Es importante para Derrida mostrar la íntima conexión de la cuestión evocada con el problema general de la diferencia ontológica: contar historias -es decir, pretender, como lo hacía Hegel, construir una estructura de inteligibilidad metahistórica de dominación de los hechos- es lo mismo que "asimilar el ser al ente" (ibid., p. 61), desconocer la diferencia ontológica, esto es, perder la diferencia entre lo que se intenta contar y el cuento mismo (o si se prefiere, entre la vivencia y el saber de esta vivencia). En Hegel se asume una indiferencia entre estas dos dimensiones (lo real es racional) y con esto se realiza una simplificación que es también una falsificación o una mentira, en el sentido antes evocado. O, como dice Derrida, se produce ahí un "discurso mitológico":

«Contar historias», entonces, a saber, abandonarse a un discurso mitológico, es algo al que se ha querido renunciar por primera vez en filosofía en el momento preciso en que el problema del ser se ha anunciado como tal [Raconter des histoires", donc, c'est-à-dire s'abandonner à un discours mythologique, c'est quelque chose à quoi on a voulu renoncer pour la première fois en philosophie au moment précisément où le problème de l'être s'est annoncé comme tel] (ibid., p. 64).

El mito aludido aquí es el de la racionalidad todopoderosa que en cambio el problema del ser, y de su indecibilidad, obligaría a suspender. Es imprescindible para Heidegger salir del vínculo de la narración, interrumpir el juego impuesto por la metafísica, y que ha hecho de Hegel el "novelista de la metafísica [romancier de la philosophie]" (ibid., p. 73), y encontrar un método que sea capaz de acceder a la historia sin pretender objetivaciones totales (o, lo que es lo mismo, es necesario acceder a la vivencia sin pretender un saber pleno de la vivencia misma, capaz de contenerla completamente). 
Es entonces el proyecto entero de "Ser y tiempo" el que debería llevar a cabo esta tarea: el método de la analítica, presentado ya en el $\S 7$, se vuelve explícitamente a este objetivo. Pero más en general aún, esto se podría ejemplificar bien al considerar la dimensión hermenéutica de la facticidad: debemos pensar la historicidad del Dasein y no del ser. O sea, no se puede llegar a pensar las estructuras de la temporalidad del ser por sí solas, porque sino se reduce el ser a un ente, pero sí se puede, jugando virtuosamente con la circularidad hermenéutica, determinar estas estructuras a partir de la problematización del ente-Dasein fáctico. El método de "Ser y tiempo" prevé precisamente que partamos del Dasein en su cotidianidad media para poder definir, en el "círculo" de su dimensión mundana, lo que la estructura.

\section{Siempre ya}

Como se ha dicho, según Heidegger habría dos modelos opuestos o dos figuras antitéticas de la historicidad: la primera figura o el primer modelo cree (o hace creer) que sabe "contar historias", y piensa la historia desde la transcendencia del cuento (es el espectro de Hegel según el cual, como se ha dicho, en la historia es el espíritu mismo el que busca maneras para manifestarse), y este modelo se mantiene en la sincronía de un presente eterno ${ }^{3}$ (perdiendo así la dinámica vivencial del acontecer); la otra figura de la historicidad sería en cambio capaz de dar espacio a, o de hacerse cargo de, la diacronía (la diferencia del ser y del ente) y con esto lograría hacer dos cosas al mismo tiempo: se refiere al ente concreto e histórico (el Dasein), y, a la vez, llega a tocar la esencia y el sentido del fluir histórico (esta sería la nueva comprensión esencial de la historia buscada por Heidegger en el capítulo cinco y la apertura al tema de la temporalidad del ser que se tenía que realizar en la sección ausente de "Ser y tiempo"). Pero entonces, a partir de un estudio de la historicidad del Dasein, Heidegger suponía poder encontrar un punto de conjunción de dos tendencias opuestas: la contingencia siempre condicionada de los hechos concretos y fácticos, y la transcendencia de un acceso a lo incondicional temporal: el acontecer como tal.

Ahora bien, frente a este intento, Derrida, en su curso del 1964-65, no podría ser más elogioso. Baste con citar las páginas muy densas en las que Derrida valoriza el rol que juega, en el planteamiento de "Ser y tiempo", la expresión, recurrente en el texto, de "siempre ya" (immer schon). Tal expresión, 
que suena como algo estridente y que sin embargo se ha impuesto en la jerga heideggeriana y no solo, es utilizada por Heidegger para calificar la condición existencial mínima del Dasein en su estar en el mundo. "Siempre" que el Dasein emprende algo, lo ha emprendido "ya": el Dasein se encuentra siempre ya en el mundo, siempre ya diseminado en una preocupación óntica, y siempre ya con los otros de quienes no se separa. El "siempre ya" juega así, en la analítica existencial, el papel contrario al per se. El Dasein es diseminado al mundo, porque es "siempre ya".

Derrida subraya el hecho que en esta expresión ("siempre ya") ya encontramos todo el problema de la historicidad: el "ya" indica un elemento acontecido, factual. Algo ya pasado. El "siempre", en cambio, dice Derrida, supone una temporalidad que puede superar la contingencia en una continuidad sin inicio y sin fin ("siempre"), y por esto afuera de la historia. ${ }^{4}$ Así, comenta Derrida,

el «siempre» modifica el ya de tal manera que el ya no depende de tal o tal otra situación contingente sino que adquiere un valor de universalidad «incondicionada». El siempre arranca la historicidad del ya a la empiricidad [Le «toujours» modifie le déjà de telle sorte que le déjà ne dépend pas de telle ou telle situation contingente mais ait valeur d'universalité «inconditionnée». Le toujours arrache l'historicité du déjà à l'empiricité] (Derrida, 2013, p. 77).

O sea, sería gracias a la duplicidad nombrada por la expresión "ya siempre" que Heidegger construye y mantiene una tensión filosófica que Derrida quiere valorar e indicar como lo más propio del pensamiento heideggeriano: el "ya" no es solo empírico, el "siempre" no es solo estructural, porque el uno reenvía al otro, y el uno saca al otro la limitación de su concepto, permitiendo la circularidad hermenéutica que define el intento heideggeriano.

Derrida considera entonces que, en la circulación que se define entre el "ya" y el "siempre", se cumple la comprensión profunda de la temporalidad heideggeriana. Afirma Derrida en un momento:

La circularidad es la historicidad, o sea la gravedad de un ya ahí que pesa y da su lugar, su centro, a la cuestión del ser que ha siempre ya empezado a provocarnos, que nos sorprende [...] porque no disponemos de ella, porque ella ha siempre ya empezado, porque no podemos evitarla, porque somos tomados por ella [...]. Las contradicciones son

4 Es cierto que en “"Ser y tiempo"” Heidegger utiliza también, y en proporción parecida, la expresión je schon, que en lugar del "siempre" (immer) hace alusión al "cada vez" (je). "Cada vez" no excede la contingencia, sino que la define en la repetición no universalizable de las veces, contradiciendo en cierto modo el propósito derridiano. Pero de toda forma, no obstante esta variación expresiva, el sentido general de la lectura de Derrida no está en discusión. 
la historicidad, es decir, la imposibilidad de un punto de partida puro en la proximidad absoluta de lo óntico o de lo ontológico [La circularité est l'historicité, c'est-à-dire la gravité d'un déjà là alourdissant et donnant son lieu, son centre, à la question de l'être qui a toujours déjà commencé à nous provoquer, qui nous surprend [...] parce que nous ne disposons pas d'elle, parce qu'elle a déjà commencé, parce que nous ne pouvons pas la contourner, parce que nous sommes pris en elle [...]. Les contradictions sont l'historicité, c'est-à-dire l'impossibilité d'un point de départ pur dans la proximité absolue de l'ontique ou de l'ontologique] (ibid., pp. 142-3).

La circularidad que se instala entre el "ya" y el "siempre", una circularidad que no se resuelve en ninguno de los opuestos y que, supuestamente, no se puede tampoco sintetizar, manteniéndose al nivel de una "contradicción", sería, según Derrida, lo más propio de la historicidad heideggeriana: la imposibilidad de un punto de partida puro.

Este círculo que no se cierra, es lo que más le interesa a Derrida. Es de hecho en un sentido análogo, aunque con una terminología bastante distinta, que Derrida volverá sobre la misma valorización de la circularidad heideggeriana en el texto "Dar el tiempo", de 1991 (es decir, 25 años más tarde). Dice ahí Derrida:

Habría más que decir acerca de la figura del círculo en Heidegger. Su tratamiento no es sencillo. Implica también una cierta afirmación asumida del círculo. La circularidad no debería necesariamente ser rehuida o condenada, como lo sería una mala repetición, un círculo vicioso, un proceso regresivo o estéril. Es preciso, en cierto modo, por supuesto, habitar el círculo, dar vueltas en él, vivir en él una fiesta del pensamiento; y el don, el don del pensamiento, no sería ajeno a esto (Derrida, 1995, p. 20).

Hasta aquí entonces, parece que Derrida quisiera suscribir sin excepciones todo el movimiento heideggeriano y todo el planteamiento de la historicidad propuesto en "Ser y tiempo".

\section{Pero ya no}

Sin embargo, Derrida no deja de plantear, justo en ese punto, sus críticas. No es una crítica la suya, en realidad, por la misma razón por la cual no es una "crítica", en general, la deconstrucción: ${ }^{5}$ Siempre se trata más bien de contaminar

5 Cf, Derrida, 1992, p. 60: "la déconstruction n'est pas une opération critique, le critique est son objet ; la déconstruction porte toujours, à un moment ou à un autre, sur la confiance faite à l'instance critique, criticothéorique c'est-à-dire décidante, à la possibilité ultime du décidable ; la déconstruction est déconstruction de la dogmatique critique". La apuesta aquí no es secundaria: si la crítica se entiende como un trabajo racional de objeciones a una tesis definida, en vista de realizar una transparentación progresiva de sus presupuestos, en un camino dirigido, aun sea asintóticamente, hacia la verdad (se pueden oponer otras objeciones a la primera, en vista de avanzar y salir del conflicto -asumiendo entonces un "afuera" del conflicto), en cambio la deconstrucción no procede por oposición de tesis, no procede siquiera por "posiciones", ni por la idea de 
las palabras de los filósofos con una indecidibilidad, se trata de habitar los textos y de llevarlos al límite de su incertidumbre o de un imposible cierre (del imposible cierre del círculo, justamente). Derrida muestra una duplicidad de intentos en obra en el texto heideggeriano. Si, por un lado, Heidegger valoriza este círculo que no se cierra, la inadecuación del siempre al ya y del ya al siempre, que mueve el uno hacia el otro, por otro lado, Heidegger mantendría, en el esquema de su argumento, una orientación resolutiva (un deseo de cerrar el círculo). Heidegger terminaría expresando en sus análisis un deseo de encontrar una "unidad de totalización [unité de la totalisation]" (Derrida, 2013, p. 206) del problema histórico. Emergería en suma en Heidegger la exigencia de una suerte de síntesis que, por cuanto no recaiga en el horizonte de una consciencia o de un cogito, llevaría en todo caso consigo el espectro de un presente pleno, no-histórico, no distinto del "presente vivo" husserliano o del devenir histórico hegeliano.

Si en Husserl el presente vivo es la expresión de una búsqueda de transparencia teórica o la forma universal e incondicionada de la experiencia pura, la forma última y sin reenvíos; y si en Hegel no hay historia sin la posibilidad de una trascendencia racional que la gobierne y que pueda transparentarse a sí misma; de modo, por fin, no tan diferente, ${ }^{6}$ Heidegger busca un horizonte unitario del acontecer en la historicidad auténtica, desconociendo en este sentido, como dice Derrida, que

mientras más el problema de la historicidad sea reconducido a su arraigo [enracinement], es decir, al problema de la temporalidad, más los medios categoriales y la seguridad de un horizonte se pierden [Plus le problème de l'historicité est reconduit à son enracinement c'est-à-dire au problème de la temporalité, plus les moyens catégoriaux et de sécurité de l'horizon se dérobent] (Derrida, 2013, p. 241).

O sea, Heidegger tenía que reconocer que, al radicalizar su propósito, las rasgos de la historicidad se hacían menos claros y definidos; en cambio, el solo hecho de que Heidegger haya puesto el argumento de la historicidad del Dasein al

una elección entre ellas, porque se trata más bien de un trabajo vuelto a mostrar la contaminación de los términos que las componen: no se trata de buscar racionalmente un remedio al error, porque el remedio es el veneno y porque cada veneno es en cierta dosis el justo remedio. La operación buscada no es entonces la de transparentar en vista de encontrar un punto de lucidez y de oponerlo a otra mirada. La deconstrucción deconstruye en primera instancia la facilidad de las distinciones y de los contrastes críticos.

6 Puede chocar la asociación del gesto heideggeriano sobre la historicidad a Hegel, pero para el Derrida de unos años más tarde, parece quedar perfectamente claro que no obstante Heidegger "se querría seguramente no hegeliano", en realidad "la historicidad es inmediata y esencialmente determinada como espiritual" (Derrida, 1987, p. 61). Lo cual se hace evidente, según Derrida, por el hecho que Heidegger ya desde los textos que siguen a "Ser y tiempo" empiece a utilizar conjuntamente los adjetivos geistig y geschictlich, asociándolos con un guion: gestig-geschictlich. 
servicio de una tercera sección de "Ser y tiempo" que -aunque ausente- anuncia que se estudiará la temporalidad del ser, implica cierto deseo de superar la falta de horizontes y la duplicidad del siempre ya. Heidegger no se limitaría ahí a la imposibilidad de un pensamiento unitario de la historicidad, sino que estaría en cierto modo ya siempre a punto de hacer de esta imposibilidad el rasgo de una nueva comprensión total de la historia.

Dicho con las palabras de la última (y enigmática) página del curso de Derrida, Heidegger terminaría necesitando en "Ser y tiempo" un lenguaje metafórico, o sea capaz de hacerse alusivo y de indicar eso que escapa a toda determinación gramatical (el Ser). Pero, observa críticamente Derrida (y cierra el curso con esta afirmación): "lo que se esconde debajo de esta otra metáfora es la apertura de la cuestión misma, es decir, de la diferencia [Ce qui se cache sous cette autre métaphore, c'est l'ouverture de la question elle-même, c'està-dire de la différence]" (Derrida, 2013, pp. 325-326).

O sea, detrás del argumento ontológico, y detrás del deseo heideggeriano de abrir la tercera sección sobre la temporalidad del ser, en realidad emerge el problema más profundo de la diferencia como tal (que Heidegger no habría sabido plantear completamente).

¿Cuál sería el problema entonces? La diferencia es en Heidegger ontológica, es decir, es diferencia del ser con el ente, y hablar del ser, aunque sea alusivamente, metafóricamente, implica un deseo de "determinarlo" (o sea, de liberarlo de la diferencia). Heidegger plantearía su pregunta y el tema de la diferencia, siempre y solo en vista de acceder a lo que difiere. Se pregunta a ese respecto Derrida en la misma última página:

\footnotetext{
¿Determinarlo en qué? Y bien, por ejemplo, determinarlo por medio de una determinación lingüística a la cual no podemos no reenviar. Y en esta determinación lingüística queda aún una determinación a través del presente, a través de la presencia del presente, en el mismo momento en el que, en nombre de la cuestión del ser, se destruye la dominación de la presencia [Le déterminer en quoi ? Et bien par exemple, encore par la détermination linguistique à laquelle essentiellement on ne peut pas ne pas faire appel. Et cette détermination linguistique reste encore une détermination par le présent, par la présence du présent, au moment même où au nom de la question de l'être on détruit la dominance de la présence] (ibid., p. 324).
}

Dicho de otro modo, Heidegger, aunque quiera destruir la dominación de la presencia (la historia de la metafísica), al solo querer cerrar el círculo de su argumento en la cuestión del ser y al generar para ese fin una metaforicidad alusiva, terminaría contradiciendo de hecho lo que primero había observado y pretendido. 
¿Y cuál sería la contradicción finalmente? El hecho de que la metáfora es un nuevo cuento. Es una historia que uno se cuenta. Heidegger no saldría entonces de este círculo: vuelve, él también, a contar historias. Heidegger había querido "destruir las metáforas" (ibid., p. 325) y toda conceptualización de las historias mentirosas, como las de Hegel, pero lo habría hecho por fin con otra metáfora, es decir, contándose otra historia: la historia del ser auténtico y puro, más allá de todo ente. Al ver la complejidad de la historicidad, y al asociar a esto el anuncio una comprensión venidera de la historicidad del ser, Heidegger contaminaría la historicidad diferencial y fáctica, el siempre ya, con un siempre puro y simple.

\section{Ya y no todavía}

Una palabra más para concluir y entender el gesto soterrado en el argumento de Derrida. ¿Qué quiere sugerir Derrida con su argumento crítico? ¿A qué está apuntando su análisis? Al hecho de que el problema está en el modo de preguntar. $\mathrm{O}$ sea, el modo en que Heidegger se dirige al problema o el esquema encontrado para responder, hace que se busque siempre evitar los riesgos de una comprensión inauténtica, salir de la narración, parar de contar historias o de mentirse. Pero para Derrida, sencillamente, no se puede suspender la ficción, no se puede parar de contar historias. No existe un punto de distinción definitivo entre cuento mentiroso e historia, entre narración y fundación. No hay manera de escapar a la ficción. Hablar es ya jugar con la diferencia, o sea, estar en la metáfora. No hay salida a esto, no hay afuera de la metafísica. Y por esto, en vez de salir o de encontrar una solución a la dominación mentirosa, se trata más bien de hacerse cargo del círculo interrumpido y de la imposibilidad de salir del siempre ya. En esto se abre un espacio para otra comprensión y otro movimiento (la deconstrucción en su conjunto). Como Derrida dirá unos pocos años después del curso sobre Heidegger, en "La escritura y la diferencia" (1972): "La diferencia no pertenece simplemente ni a la historia ni a la estructura" (Derrida, 1989, p. 44).

La diferencia precede esta oposición o la abre, y no hay forma de resolver el círculo ni de cerrarlo.

\section{Bibliografía}

DERRIDA, J. “Marges de la philosophie”. Paris: Les éditions de Minuit, 1972. . "De l'esprit. Heidegger et la question”. Paris: Galilée, 1987. "La escritura y la diferencia". Trad. P. Peñalver, Barcelona: Anthropos, 1989. 
. "Points de suspensions". Paris: Galilée, 1992.

. "Dar (el) tiempo. La moneda falsa". Trad. C. De Peretti. Barcelona: Paidós, 1995. . "Heidegger: la cuestión del Ser y de la Historia". Paris: Galilée, 2013.

GRATTON, P. "The Spirit of the Time: Derrida's Reading of Hegel in the 1964-65 Lecture Course". CR: The New Centennial Review, Vol. 15, Nr. 1, pp. 49-65, Spring 2015. HEIDEGGER, M. "Ser y tiempo". Trad. J. E. Rivera. Santiago de Chile: Editorial Universitaria, 1997.

HUGO, V. "Toute la lyre". Paris: Hetzel, 1888.

HUSSERL, E. "Ideas relativas a una fenomenología pura y una filosofía fenomenológica". (Vol. I). Trad. J. Gaos, México/Buenos Aires: Fondo de cultura económica, 1962.

NAAS, M. "Violence and Historicity: Derrida's Early Readings of Heidegger". Research in Phenomenology, Vol. 45, Issue 2, pp. 191-213, 2015.

NIETZSCHE, F. "Segunda consideración intempestiva". Trad. J. Etorena. Buenos Aires: Ed. Zorzal, 2006. 